\title{
Development and Analysis of an Information Security Learning Method using Situation-based Problems
}

\author{
Yungyeong Ko ${ }^{1}$, Seonghee Lee ${ }^{1}$ and Seong Baeg Kim ${ }^{1, *}$ \\ Dept. of Computer Education, Jeju National University, \\ Jeju-do, Republic of Korea \\ \{dbsrodzzz,heehee_2\}@hanmail.net,sbkim@jejunu.ac.kr
}

\begin{abstract}
As information appliances becomes popular and activities to bring security issues using them increase, information security is drawing much attention. However, most people hardly ever take proper steps about security problems. In order to enhance security awareness, we made a research on the information security learning, proposed a novel approach to information security learning using situation-based problems, and measured the responses of learners to the problems. We proposed how to provide a customized learning and developed the corresponding information learning contents to bring tailored learning. Finally, we examined how learners feel about information security and whether the necessity of information security learning increases or not
\end{abstract}

Keywords: Information Security Awareness, Situation-based Problem, Learning, Tailored Learning

\section{Introduction}

As computers become commercialized and widely used, negative aspects such as information leaks, improper use, game addiction have been emerged. However, the more the situations go on, the more people, who do not work for information technology, have tendency to avoid it rather than break through it or ignore the necessity of computer science education including information security education [1]. Therefore, it is required to change the misunderstanding for computer science education that includes information security education.

Education can change the awareness of human [2]. When they encounter a problem, they can solve the problem properly through the corresponding education as long as the problem is related to the awareness. We aim to change the awareness of people using proper information security learning contents by developing scenario-based problems.

Therefore, we proposed an approach to show that such misunderstanding for computer science education can be changed by education. First of all, we designed an experiment to enhance the necessity of computer science education through education, based on our previous work [8]. Due to the wide range of information technology field, we limit the scope of the experimental contents to the field of information security which is emerging recently.

The rest of the paper is organized as follows. Section 2 shortly describes the previous work related to information security education. Section 3 presents the situation-based scenarios and problems we have created and developed. Section 4 shows our

* Seong Baeg Kim (sbkim@jejunu.ac.kr) is the corresponding author of this paper. 
implementation of the customized security learning contents that corresponds to each situation-based problem. Section 5 analyzes and explains the experimental results. Finally, Section 6 makes a conclusion of the paper and suggests further study.

\section{Related Work}

In theoretical background, we show the current status of computer science education and the negative awareness. Suggesting the importance of computer science education, we have an appropriateness, which is why we choose the topic, information security education among many parts of computer science education.

\subsection{Awareness of Information Security}

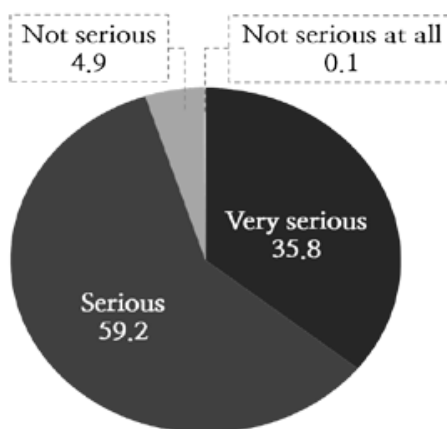

(a) Degree of dysfunctional information

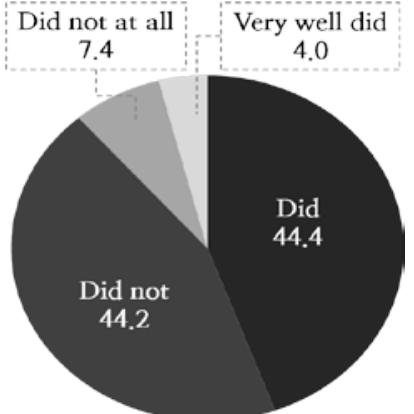

(b) Whether to take information protection measures

Figure 1. Analysis of Awareness of Information Security [3]

As we can in Figure 1 (a), $95.0 \%$ ('Very serious' 35.8\% + 'Serious' 59.2\%) of individual internet users thought that the damage caused by dysfunctional information is serious on society as a whole. As shown in Figure 1 (b), $48.4 \%$ ('Very well did' $4.0 \%$, 'Did' $44.4 \%$ ) of individual internet users answered they take action to prevent the damage, $44.2 \%$ did not.

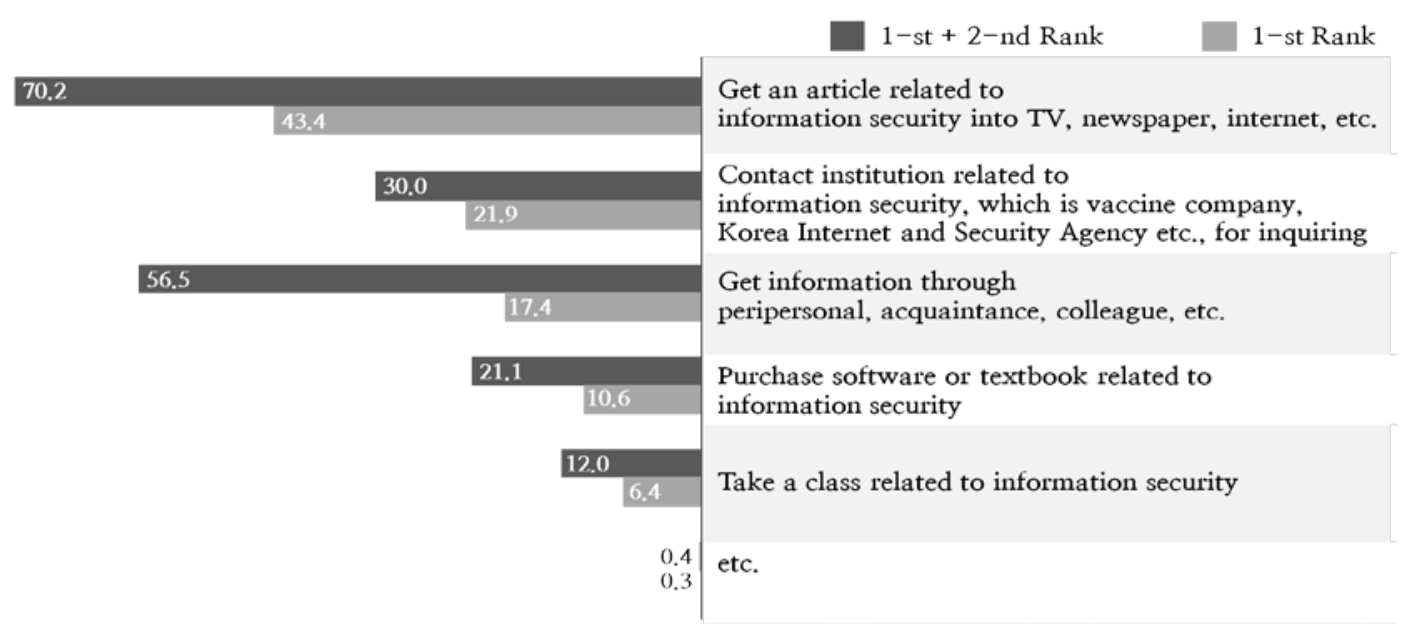

Figure 2. Types of Collecting the Knowledge of Information Security [3] 
As shown in Figure 2, about two thirds (65.3\%) of personal internet users collect upto-date information or measure about information security. In case of collection of upto-date information/measure about information security, 'get an article related to information security into TV, newspaper, internet, etc.' is most popular opinion which is 43.4 percent and there is 21.9 percent opinion to 'contact institution related to information security, which is Vaccine Company, Korea Internet and Security Agency etc., for inquiring'. The survey shows that 'purchase software or textbook related to information security', and 'take a class related to information security' are low ratio relatively [3].

\subsection{Related Research}

There was a study on a secure access to educational resources [4]. It focused on proposing an educational web model that is not related to information security education. Most of the security-related research consisted of the detailed description and theoretical knowledge about security issues [5, 6].

The case of making questions based on scenario can be found in information ethics. In the field of information ethics, there was a study that selects some issues from information ethics and then makes each questions based on the scenario [12]. However, they couldn't find out the effects because they didn't make a concrete experiment to a real group.

There has been research on the necessity and importance of computer science education [9-11]. However, they didn't connect the importance and necessity of computer science education with information security education.

There was also a study on the development of an examination tool based on a scenario to measure the cognition of information \& communication ethics [7]. However, there was high potential for student to become bored during the examination because the scenario was too much long.

Up to now, in the information security field, we couldn't find the case of developing question, based on the scenario. In the case of question making in the field of information ethics, we feel the lack of development of digital contents which can educate and evaluate the level of awareness.

Accordingly, we're going to enhance the needs of computer science education by suggesting problem situation based on the scenario of information security, arousing people's level of consciousness, and accomplishing the proper education. Also, we're going to make this situation into digital contents, apply to high school students and analyze it. It has not been made to develop digital contents for the developed questions. Also, it was insufficient that there is only the measurement of the level of awareness without contents to teach.

\section{Situation-based Scenarios and Problems}

We choose the core keywords about information security and plot the problem situations related to each keyword. We create a few situation-based problems for each keyword and present them to users. After solving the problems, users can be diagnosed on what part they lack in the information security. Based on diagnostic results, users can learn only their weak parts. After learning the weak parts, users check out whether their information security level is improved through another situation-based problems once again. The final goal is to let users to make up for their weak parts and improve the necessity of information security education through the questionnaire. 
Figure 3 shows the tailored learning flowchart. As you can see in the figure, as a first step, a learner can select a randomly selected situation-based problem and then solve it. If the learner answers it correctly, the learner doesn't have to learn the information security learning contents about the topic corresponding to the situation given. Otherwise, it is required for the learner to learn the learning contents to make up for his/her weak parts.

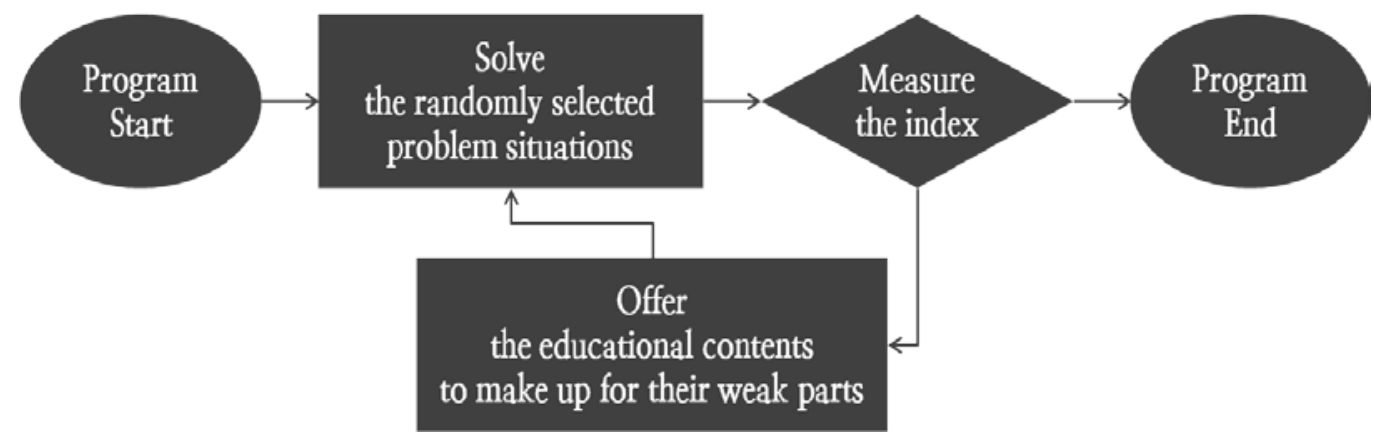

\section{Figure 3. Tailored Learning Flowchart}

We make a total of 15 situation-based problems. We make the scenarios closely related to the real life as far as possible. Thus, we draw users' high interest. Also, we can find out how each keyword is involved in each problem since we make each problem considering the relation between problems and keywords as shown in Table 1.

Table 1. Operational Definition about each Keyword

\begin{tabular}{|c|}
\hline Phishing \\
\hline - deceives users and steal their personal information. \\
\hline Spyware \\
\hline $\begin{array}{l}\text { - is installed on without the user's permission. } \\
\text { - steals the huge amounts of personal or public information. }\end{array}$ \\
\hline Virus \\
\hline $\begin{array}{l}\text { - destroys the computer. } \\
\text { - reproduces itself. } \\
\text { - affects a system. } \\
\text { - has the properties spread. }\end{array}$ \\
\hline Spam \\
\hline - sends an e-mail a user doesn`t want. \\
\hline Personal Information Leak \\
\hline $\begin{array}{l}\text { - is that the information is handled by someone except for permitted users. } \\
\text { - covers the extent to users are interested in information security. }\end{array}$ \\
\hline Hacking \\
\hline $\begin{array}{l}\text { - aims at destructing computer systems. } \\
\text { - has an access privilege which is not right. } \\
\text { - shares an expertise technically. }\end{array}$ \\
\hline
\end{tabular}




\subsection{Keyword Sampling}

In 'Research on the Actual situation about Information Security' released by KISA (Korea Internet \& Security Agency) in 2011, the types were divided as shown in Figure 4. Based on this, we were able to extract the 6 kinds of keywords. Also, we made an operational definition about each keyword.

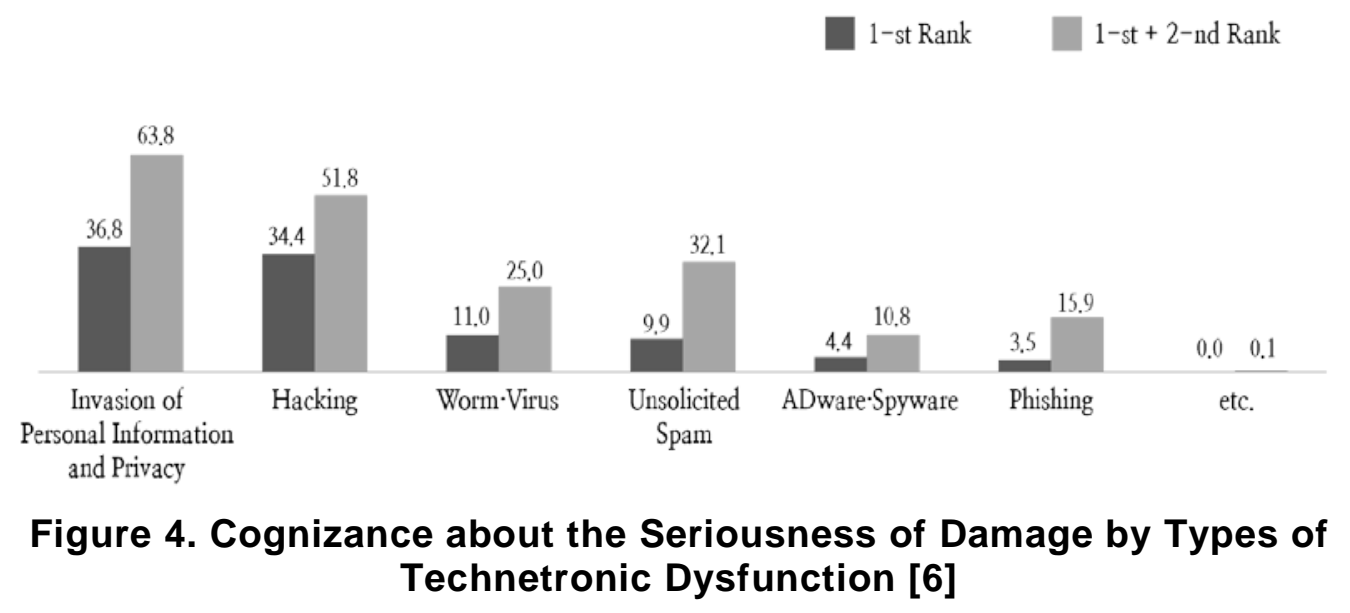

\subsection{Developing Problem Situations}

We made a total of 15 problem situations. We made the problem situations highly related to the real life as far as possible; thereby we made user raise intensity and have interest.

Table 2. Example of Problem Situation - personal Information Leak

\section{Personal Information Leak}

Jeongim, who is middle school senior, has used identical user ID and password since childhood. The user ID and password for all the sites are identical because she can't break the rules whenever she joins a new site. But nowadays, whenever she logs in, the page that recommends changing the password appears repeatedly. It is not difficult to change password, but she wanted to maintain the rules that have kept since she was a kid. In this situation, what would you do if you were Jeongim?

(1) She once the change password and converts passwords of other websites to the same password to keep her rules.

(2) She changes and manages password using the tools like passphrase.

(3) She shuts off the browser because the page might be a hacked page.

(4) It is better for her to use the password without change.

(5) I don't know.

\subsection{Level Measurement Method}

If we analyze a problem situation, we can what kind of keyword is involved in each problem situation, as shown in Table 3 . When we measure the index, we classify the problems, which include many keywords per problem situation, into harder problems. Thus, the problem, with many keywords, is put on a weigh. 
Table 3. The Relation between Problem Situation and Keyword

\begin{tabular}{|c|c|c|c|c|c|c|c|c|c|c|c|c|c|c|c|c|}
\hline \multicolumn{2}{|r|}{ Problem situation } & 1 & 2 & 3 & 4 & 5 & 6 & 7 & 8 & 9 & 10 & 11 & 12 & 13 & 14 & 15 \\
\hline \multicolumn{1}{|c|}{ Keyword Phishing } & & & & $\mathbf{O}$ & & $\mathbf{O}$ & $\mathbf{O}$ & $\mathbf{O}$ & $\mathbf{O}$ & & & $\mathbf{O}$ & & & \\
\hline 1 & Spyware & & $\mathbf{O}$ & & & $\mathbf{O}$ & & & & & & & & & & \\
\hline 2 & Virus & & & & & & & & & & & & & & $\mathbf{O}$ & $\mathbf{O}$ \\
\hline 3 & Spam & & & & $\mathbf{O}$ & & & & & & $\mathbf{O}$ & & & & & \\
\hline 4 & Personal Information Leak & $\mathbf{O}$ & $\mathbf{O}$ & $\mathbf{O}$ & & & & & $\mathbf{O}$ & $\mathbf{O}$ & & $\mathbf{O}$ & & $\mathbf{O}$ & & \\
\hline 5 & Hacking & & & & & & $\mathbf{O}$ & $\mathbf{O}$ & & & & & & & & \\
\hline 6 & &
\end{tabular}

Also, we can found out which keyword is more incorrect among the problems solved. Because there is a possibility that users don 't know a particular keyword even though they have a high index, we analyze what keyword is wrong intensively on particular keyword.

\subsection{Developing Contents for Education}

An educational content is developed as six videos for each keyword. The videos are produced, based on scenarios and covered by definition, related term, type, example, symptom, and prevention, etc. for each keyword.

\section{Implementation}

We state the six problem situations related to the six keywords. Then we show the test result and tailored learning contents corresponding to it. After making users learn the contents, we state new problem situations to them and measure their responses again. We implemented the system using HTML, PHP and JavaScript, as shown in Figure 5. 


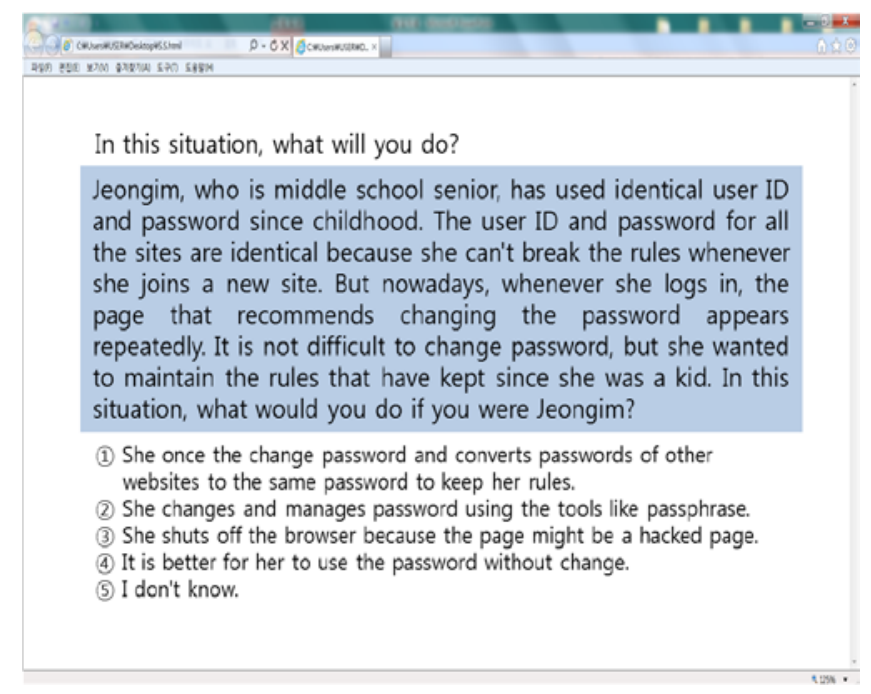

Figure 5. Video-type Learning Contents

\section{The Experimental Results}

The Korean students in the survey were between the ages of 17 and 26. The number of students sampled is 50 in total. We investigated general high school and university students randomly.

\subsection{The Survey of Content}

Among the respondents, in Figure 6 (a), a high level of 42 percent (Very interested $10 \%$, Interested $32 \%$ ) said they are concerned about information security. The proportion of those who thought 'Normal' was 42 percent. That was not low proportion. According to the survey, in Figure 6 (b), the media's impact (News 34\%, News accounts $32 \%$ ) was a big reason among the reasons that become to have interest in information security. There were other comments, 'taking the classes', 'having a personal experience', etc.
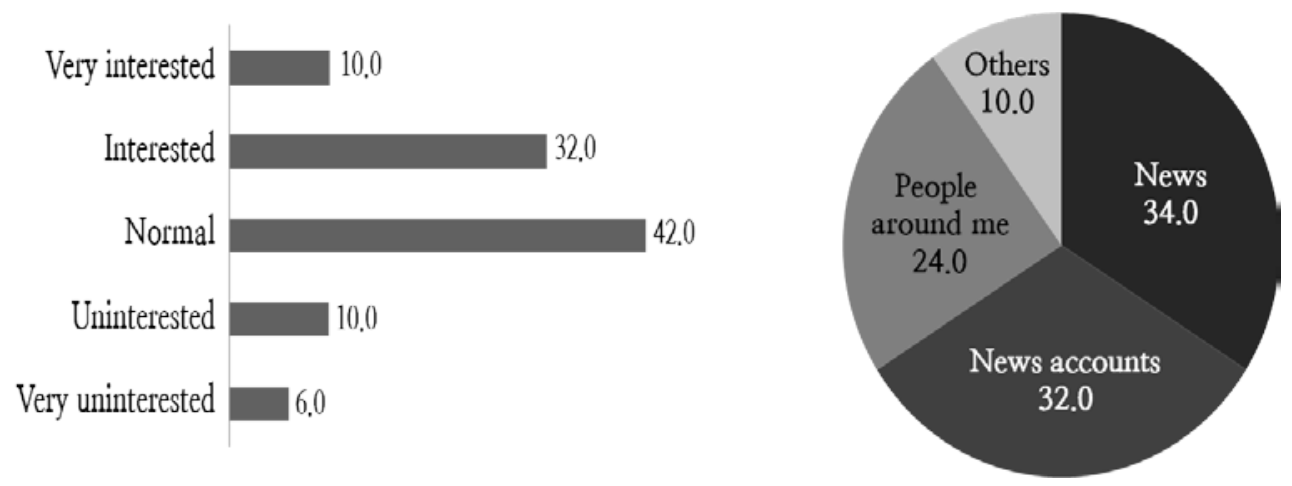
(a) Degree of interest
(b) Types of chances to have interest

\section{Figure 6. Analysis of Interest}




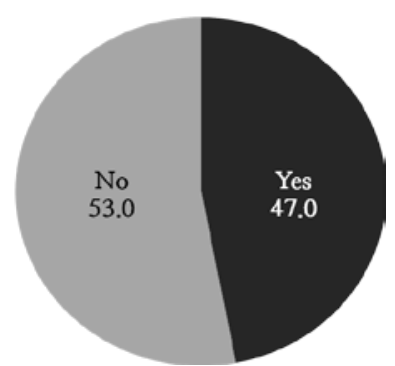

(a) Trouble

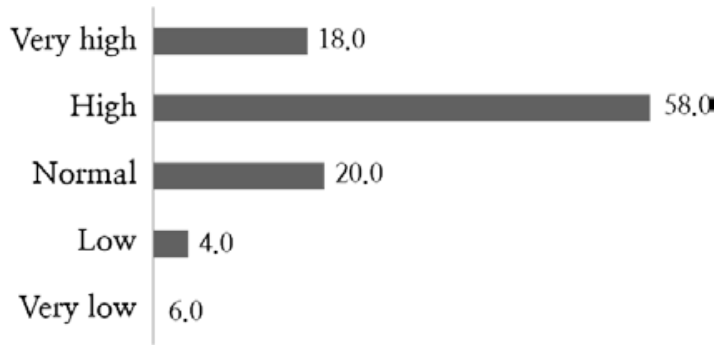

(c) Educational effects level

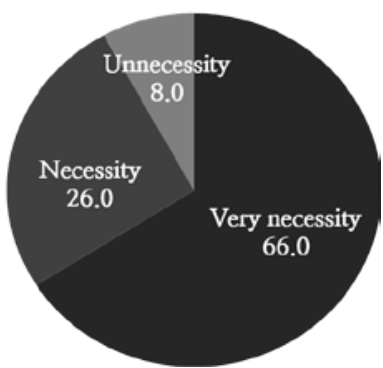

(b) Necessity

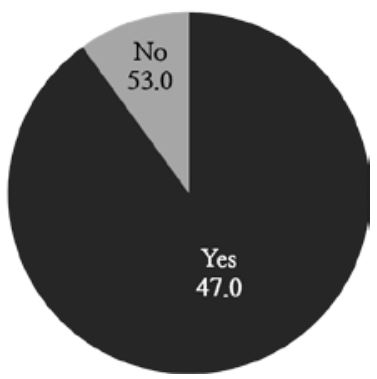

(d) Sympathy

Figure 7. Analysis of Learning Effects

Among the students surveyed, in Figure 7 (a), the ratio that got in trouble due to information security was 47 percent close to 53 percent who was inexperienced. In Figure 7 (b), 66 percent of the students surveyed recognized the necessity of computer science education. In Figure 7 (c), 76 percent of the students surveyed thought that the contents have highly educational effects. There were also minor comments (4\%) that the educational effects are low. After learning the contents, in Figure 7 (d), 90 percent of the students surveyed make sympathy with the necessity of computer science education.

After learning contents, having unsympathizing attitude about the necessity of computer science education

- After learning contents, raising the necessity of computer science education

- The level of interest about information security
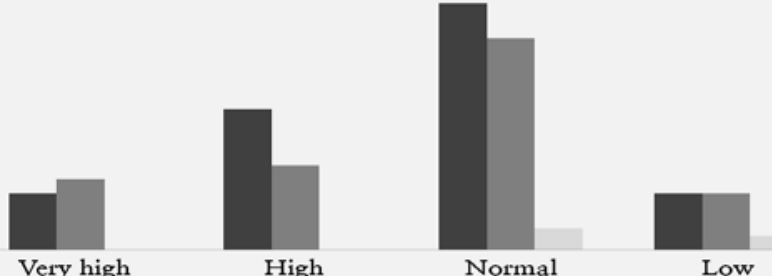

Very low

Figure 8. The Relation between the Level of Interest and Sympathy about the Necessity of Computer Science Education 
Among the students surveyed, the more interested usually in information security, the more sympathized with the necessity of computer science education after learning the contents, as shown in Figure 8.

\section{Conclusion}

After we formulated a few problem situations related to information security, and diagnosed a users` level using them. We made a users learn information security using tailored learning contents. Then through the questionnaire, we asked users that they feel the necessity of computer science education.

We made a few situation-based problems related to information security, and diagnosed a user's security cognition level using them. Then, we made a user learn tailored learning contents for information security, based on the diagnosis result. According to the result the questionnaire, users felt the necessity of computer science education on the whole. The more interest some people have of information security, the bigger necessity of computer science education they felt. Though some people have little interest in information security, more than $60 \%$ feel the necessity of computer science education after they learn the contents. Reflected on these opinions, we hope that computer science education will be revved up.

Further study, we plan to make a method of measuring the index in detail and study a problem considering interest.

\section{Acknowledgements}

This research was supported by the 2013 scientific promotion program funded by Jeju National University.

\section{References}

[1] J. Lee, “Like This Way of Computer Science Education”, Is It Right in School, Chungbukllbo, (2010).

[2] T. Seong, "Fundamental of Educational Evaluation”, Hakji Press, (2009), pp. 16-17, M. Kim, H. Kim and W. Lee, “The Development of Issue-Oriented Dilemmas for Measuring Information Ethical Judgments", The Journal of Korean Association of Computer Education, vol. 15, no. 4, (2012), pp. 13-24.

[3] Korea Internet \& Security Agency: 2011 Information Security Research on the Actual Condition, (2012).

[4] O. N. Qunoo and H. Hamad, "Secure Model for Educational Resources”, International Journal of Security and Its Applications, vol. 7, no. 1, (2013), pp. 31-50.

[5] J. Piao and S. B. Kim, "The Design and Analysis of a Hardware-based Anomaly Detection Scheme", International Journal of Security and Its Applications, vol. 6, no. 2, (2012), pp. 367-372.

[6] S. Song, H. Park and B. Choi, "E-LPG: Energy Efficient Location Privacy Scheme Against Global Attackers in Sensor Networks", Secure Model for Educational Resources, International Journal of Security and Its Applications, vol. 7, no. 2, (2013), pp. 27-46.

[7] S. Kim, H. Yoo, G. Kim and S. Cho, "The Development of Test Items based on Scenario to Measure Information and Communication Ethics”, The Journal of Korean Association of Information Education, vol. 13, no. 2, (2009), pp. 237-246.

[8] Y. Ko, S. Lee and S. B. Kim, "Developing the Problem Situations of Information Security and Diagnosing a User's Security Level”, Proceedings of Korean Association of Computer Education, vol. 17, no. 1, (2013), pp. 121-124.

[9] G. Kim, "Problems and Necessity of Computer Education in the Elementary, Middle and High School", Journal of the Korean Institute of Information Scientists and Engineers, (2009).

[10] E. Kim, "A Study of Algorithm Learning Methods for Improvement of Elementary and Middle School Students' Problem-Solving Abilities”, Journal of the Korea Society for Fisheries and Marine Sciences Education, (2011).

[11] H. Kim, "The Impact of ICT Use on Students`Academic Performance based on PISA 2006 Korean Data”, The Journal of Korean Education, vol. 35, no. 4, (2008), pp. 107-129.

[12] M. Kim, “The Development of Issue-Oriented Dilemmas for Measuring Information Ethical Judgments”, Journal of Korea Association of Computer Education, (2012). 


\section{Authors}

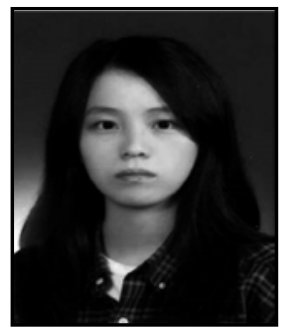

Yungyeong Ko is currently an undergraduate student of the Dept. of Computer Education at Jeju National University. Her research interests include computer science education and computer security education.

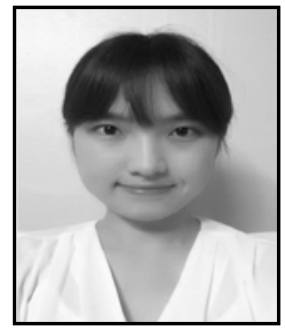

Seonghee Lee is currently an undergraduate student of the Dept. of Computer Education at Jeju National University. Her research interests include computer science education and computer security education.

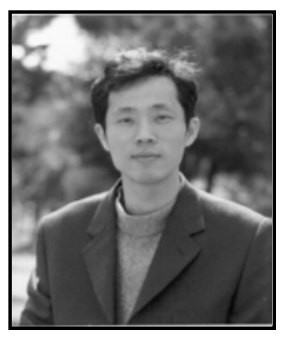

Seong Baeg Kim received the B.S., M.S., and Ph.D. in Computer Engineering from Seoul National University, Korea, in 1989, 1991, and 1995 respectively. He is currently a professor of the Dept. of Computer Education at Jeju National University, where he has been since 1996. He was a visiting scholar at Dept. of Computer Science, Montana State University from 2001 to 2002 and Dept. of Electrical \& Computer Engineering, University of Cincinnati from 2008 to 2009. His research interests include computer science education, computer system architecture, and computer security. 